\title{
Adaptation of dinitrosalicylic acid method to microtiter plates
}

\section{Cristiana Gonçalves, Rosa Maria Rodriguez-Jasso, Nelma Gomes, José A. Teixeira and Isabel Belo*}

\author{
Received 27th August 2010, Accepted 24th September 2010 \\ DOI: 10.1039/c0ay00525h
}

\begin{abstract}
A microtiter plate adaptation of the well-known dinitrosalicylic acid (DNS) colorimetric method, for measurement of reducing sugars, is described. This method allows a substantial volume reduction of the reagents and a rapid analysis of a large number of samples, having an economic cost benefit and a positive impact on the environment. Therefore, it is well suited as an high-throughput technique for reducing sugars determination.
\end{abstract}

\section{Introduction}

In 1921, Sumner introduced the dinitrosalicylic acid (DNS) method for assaying reducing sugars in normal and diabetic urine. ${ }^{1,2}$ The DNS reacts with reducing sugars, and other reducing molecules, forming 3-amino-5-nitrosalicylic acid, an aromatic compound that strongly absorbs light at $540 \mathrm{~nm}$, therefore allowing a quantitative spectrophotometric measurement of the amount of reducing sugar present.

In our laboratory, reducing sugars have been routinely measured by this method, with some adjustments to the technique, as reported in the literature. ${ }^{3-5}$ It has been observed that, besides the high volumes of sample and DNS reagent required, this methodology has the main disadvantage of including a very time-consuming step: the transfer of the reaction mixture from the digestion vessel (usually test tubes) to a new one, adequate to the absorbance measurement (cuvette or microtiter plate well). This sample manipulation is very time consuming, especially when a large number of samples are taken under consideration. Therefore, our aim was to adapt this well-known procedure, for quantification of reducing sugars, to a new one where the reaction and absorbance measurement are performed in the same vessel, in wells of a microtiter plate.

\section{Traditional method}

The DNS reagent is prepared by dissolving $5 \mathrm{~g}$ of dinitrosalicylic acid (Acros Organics) in $250 \mathrm{~mL}$ of distilled water at $80^{\circ} \mathrm{C}$. When this solution reaches room temperature, $100 \mathrm{~mL}$ of $\mathrm{NaOH}, 2 \mathrm{~N}$ (Sigma) and $150 \mathrm{~g}$ of potassium sodium tartarate-4-hydrate (Merck) are added and the volume is completed with distilled water to $500 \mathrm{~mL}$. The standard calibration curves are generally on glucose.

According to the traditional method, $500 \mu \mathrm{L}$ of dinitrosalicylic acid reagent and $500 \mu \mathrm{L}$ of sample, or distilled water (blank), are added to test tubes. The tubes are dived in a water bath $\left(100^{\circ} \mathrm{C}\right)$, for 5 minutes and then cooled in frozen water, while $5 \mathrm{~mL}$ of distilled water are added to each tube, resulting on the final reaction mixture. The addition of water while the tubes are dived in cold water is performed to stop immediately the reaction.

IBB - Institute for Biotechnology and Bioengineering, Center of Biological Engineering, University of Minho, Campus de Gualtar, 4710-057 Braga, Portugal. E-mail: ibelo@deb.uminho.pt; Fax: +351 253678986; Tel: $+351253604413$
When the tubes reach room temperature, $300 \mu \mathrm{L}$ of the resulting reaction mixture of each test tube are transferred to a well of a microtiter plate. Then the absorbance is read on a modular absorbance microtiter plate reader (Sunrise, Tecan-Switzerland), at a wavelength of $540 \mathrm{~nm}$.

\section{Development of the adapted method}

The traditional method works but, as mentioned before, it is very time-consuming when it is necessary to analyse a large number of samples. Consequently, the initial idea was to proportionally reduce the original volumes so that the reaction could occur directly on the microtiter plate-well, instead of occurring in the assay tube. With the reduction of the reagent volume other advantages emerge. There is an obvious cost saving on chemicals but also on the treatment of the resulting mixture, since harmful chemicals are used.

The microtiter plate cannot be dived in the two traditional steps: reaction step (boiling water) and the reaction stop step (frozen water); therefore the water bath was replaced by an oven $\left(\right.$ at $105^{\circ} \mathrm{C}$ ) and the frozen water replaced by ice. Notice that some brands of microtiter plates melt at $105{ }^{\circ} \mathrm{C}$, therefore it was necessary to use resistant microtiter plates (96 flat test plate of Orange Scientific, ref. no 5530100, made of crystal polyester). The reaction was carried out in wells of $340 \mu \mathrm{L}$, adding $25 \mu \mathrm{L}$ of DNS reagent to $25 \mu \mathrm{L}$ of sample, or distilled water (blank). Subsequently, in order to perform the reaction, the microtiter plate, with cap, was placed in the oven by $5 \mathrm{~min}$, then it was placed on ice and $250 \mu \mathrm{L}$ of distilled water was immediately added to each well. According to these adjustments, the calibration curve with glucose (Fluka) was obtained (Fig. 1).

When comparing the calibration curve obtained for the adapted method with the traditional method curve, it is possible to observe a decrease in sensitivity, shown by the decrease of the slope from $0.42 \pm 0.02$ to $0.052 \pm 0.002$. This reduction in sensitivity is possibly explained by the fact that the reaction traditionally occurs on a water bath, where better heat transfer occurs than in the oven. In order to solve this problem the oven time was increased to $10 \mathrm{~min}$, providing more time for heat transfer, ensuring that the system will be at around $100{ }^{\circ} \mathrm{C}$ during the required reaction time. The calibration curve obtained with 10 min of reaction is presented in Fig. 1. The curve obtained is very similar to the curve obtained with the traditional method, performed in test tubes. For this reason no further modifications 


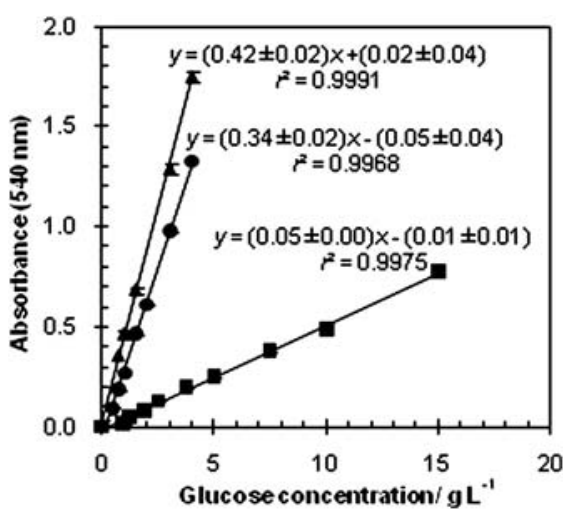

Fig. 1 Standard calibration curves for the modified DNS assay performed with 5 minutes of reaction $(\boldsymbol{\square})$ and 10 minutes of reaction $(\bullet)$, and for the traditional DNS assay -5 minutes of reaction $(\boldsymbol{\Delta})$. Results are the mean of six replicates for each concentration.

were introduced to the method and new calibration curves were performed to validate the method. Notice that the calibration curves presented on Fig. 1 are only comparable since all the absorbance measures, even the ones of the traditional method, are performed using the same reaction mixture volume $(300 \mu \mathrm{L})$, in the same absorbance reader and with the same type of microtiter plate.

The adapted method was used in fermentation samples, achieving good results even in complex media, like olive mill wastewaters (OMWs), Fig. 2. Reducing sugars of the samples, collected through time, were determined through both methods. The values obtained by the adapted method are higher than those obtained by the traditional method, especially when higher concentrations are analysed, demonstrating an improvement on the sensitivity of the method.

A partial validation of the adapted method was performed by the analysis of the following parameters: linearity and sensitivity, limits of detection and quantification and precision. These parameters were also estimated to the traditional method, allowing a full comparison between both methods. Notice that for the new method 5 independent calibration curves were considered and for the traditional one only 2 curves were considered. All statistical treatments were performed using Microsoft Excel 2007 software.

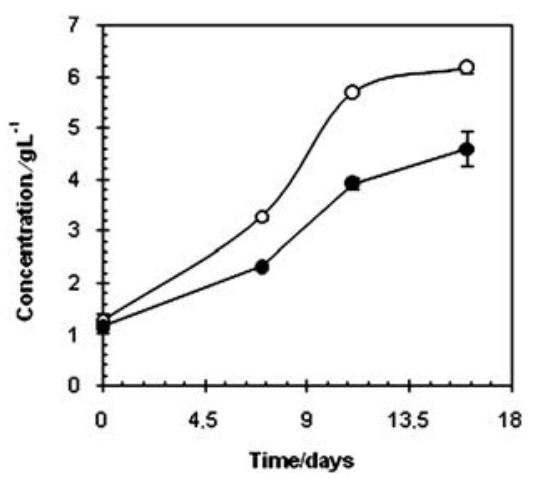

Fig. 2 Reducing sugars, through time of an OMW fed-batch fermentation, analyzed by the traditional method $(\mathbf{O})$ and the adapted method (○). Data are presented as the mean and standard deviation of three independent experiments.
Linearity is the ability of an analytical method to produce results that are directly proportional to the concentration of the analyte present in the samples, in a known range of concentrations. It was defined through the relation between glucose concentration and absorbance measured at an optical density of $540 \mathrm{~nm}$. The correlation coefficient $(r)$ was calculated by the least squares method. This parameter is frequently used to indicate the adequacy of the curve to the mathematical model. The coefficient of determination $\left(r^{2}\right)$ was also determined in order to verify how well the regression line represents the data. As the deviations from linearity are often difficult to detect visually, its suitability was checked by calculating the residues between the measured and the calculated values obtained from the regression equation, performing the $t$-test. The calibration curves obtained from the relation between glucose concentration and the absorbance values, measured at $540 \mathrm{~nm}$, by the adapted and the traditional methods, were all linear (with 95\% confidence level) for the concentration range of $0.06 \mathrm{~g} \mathrm{~L}^{-1}$ to $4 \mathrm{~g} \mathrm{~L}^{-1}$. To compare the reproducibility of the 5 calibration curves obtained for each independent assay of the adapted method (Table 1), the standard deviation between their slopes and $y$-intercepts was calculated. As a result, it was possible to confirm that these calibration curves were very similar among themselves, with a standard deviation of 0.04 and 0.01 for the slope and $y$-intercept, respectively. They were also similar to the first calibration curve obtained of the modified method with a reaction time of $10 \mathrm{~min}$ (Fig. 1), represented as: $y=(0.34 \pm 0.02) x-(0.05 \pm 0.04)$, with 0.9968 as coefficient of determination $\left(r^{2}\right)$ and 0.9984 as coefficient of correlation $(r)$. The good values obtained for the calculated $r$ and $r^{2}$ suggest a strong linear relationship between the two variables and an excellent representation of the data by the regression equations of the curves obtained. For the traditional method, the calibration curve was represented as: $y=(0.42 \pm$ $0.02) x-(0.02 \pm 0.04)$ with a coefficient of determination of 0.9991 and a coefficient of correlation of 0.996 .

The sensitivity of an analytical method is the capacity to distinguish small differences in the analyte concentration and it can be determined by the slopes obtained in the calibration curves. The sensitivity obtained from the calibration curves for both methods were quite similar, $0.34 \pm 0.02$ and $0.42 \pm$ 0.02 for the adapted and the traditional methods, respectively.

The limit of detection (LOD) is defined as the smallest concentration of analyte in the sample that can be reliably distinguished from zero and the limit of quantification (LOQ) is the concentration below which the analytical method cannot operate with an acceptable precision. These parameters were calculated from the calibration lines that defined linearity, using

Table 1 Standard calibration curves of the adapted method traced in different months

\begin{tabular}{ll}
\hline Month & Calibration curve \\
\hline 0 & $y=(0.34 \pm 0.02) x-(0.05 \pm 0.04)$ \\
2 & $y=(0.35 \pm 0.03) x-(0.05 \pm 0.04)$ \\
12 & $y=(0.31 \pm 0.01) x-(0.05 \pm 0.02)$ \\
14 & $y=(0.41 \pm 0.02) x-(0.05 \pm 0.03)$ \\
18 & $y=(0.30 \pm 0.01) x-(0.04 \pm 0.02)$ \\
\hline
\end{tabular}


eqn (1) and (2), according to the Long and Winefordner criterion. ${ }^{6}$

$$
\begin{aligned}
& \mathrm{LOD}=\left(3 \times S_{\mathrm{B}}\right) / m \\
& \mathrm{LOQ}=\left(10 \times S_{\mathrm{B}}\right) / m
\end{aligned}
$$

where $S_{\mathrm{B}}$ is the standard error of the independent term of the regression and $m$ is the analytical sensitivity. For the adapted method, the glucose concentrations of $(0.11 \pm 0.03) \mathrm{g} \mathrm{L}^{-1}$ and $(0.36 \pm 0.10) \mathrm{g} \mathrm{L}^{-1}$ were defined as the limits of detection and quantification in that order. The original method limits for glucose were, respectively, $(0.10 \pm 0.03) \mathrm{g} \mathrm{L}^{-1}$ and $(0.33 \pm 0.06) \mathrm{g} \mathrm{L}^{-1}$.

Precision is the smallest divergence between independent test results obtained under stipulated conditions, for the same sample. It is usually specified in terms of standard deviation (SD) or relative standard deviation (RSD). The precision of the method was analysed in terms of repeatability by the Horwitz criterion. ${ }^{7}$ According to this criterion, to consider RSD acceptable in terms of precision, the RSD experimental values should be lower than the RSD values calculated by the Horwitz formula (eqn (3)).

$$
\operatorname{RSD}(\%)=2^{(1-0.5 \log C)}
$$

where $C$ is the concentration expressed as powers of ten. Considering the curves obtained for the adapted method, the calculated RSD was $3.5 \%$ and the theoretical was $6.8 \%$. Therefore, considering the Horwitz criterion, the method is precise since the calculated RSD is lower than the theoretical RSD. The traditional method is also precise since the calculated RSD was $5.7 \%$ and the theoretical was $6.8 \%$.

\section{Conclusions}

The dinitrosalicylic acid (DNS) method for the assessment of reducing sugars was adapted to microtiter plates and it is suitable for routine analysis of reducing sugars. It reduces both assay time and volume of reagent (20-fold), decreasing the global cost and the hazards of the assay, allowing the analysis of several samples at once. Although the reaction time has increased to 5 minutes, the overall time of the analysis has significantly diminished. This reduction is mainly due to the fact that the reaction occurs directly on the location where the absorbance will be read, avoiding the time consuming step of transferring the reaction mixture of each sample to every single well of the microtiter plate.

\section{Acknowledgements}

The authors acknowledge Fundação para a Ciência e Tecnologia (FCT) and Mexican Science and Technology Council (CONACYT) for the financial support provided (SFRH/BD/27915/ 2006; SFRH/BD/28039/2006; Project PTDC/AMB/69379/2006; CONACYT 206607/230415).

\section{References}

$1 \mathrm{~J}$. B. Sumner, The estimation of sugar in diabetic urine, using dinitrosalicylic acid, J. Biol. Chem., 1924, 62, 287-290.

2 J. B. Sumner, Dinitrosalicylic acid: a reagent for the estimation of sugar in normal and diabetic urine, J. Biol. Chem., 1921, 47, 5-9.

3 G. L. Miller, Use of dinitrosalicylic acid reagent for determination of reducing sugar, Anal. Chem., 1959, 31, 426-428.

4 F. J. Robyl and W. J. Whelan, Reducing value methods for maltodextrins: 1 . chain length dependence of alkaline $3: 5$ dinitrosalicylate and chain length independence of alkaline copper, Anal. Biochem., 1972, 45, 510-516.

5 S. Sengupta, M. L. Jana, D. Sengupta and A. K. Naskar, A note on the estimation of microbial glycosidase activities by dinitrosalicylic acid reagent, Appl. Microbiol. Biotechnol., 2000, 53, 732-735.

6 G. L. Long and J. D. Winefordner, Limit of detection. A closer look at the IUPAC definition, Anal. Chem., 1983, 55, 712A-724A.

$7 \mathrm{~W}$. Horwitz, Evaluation of analytical methods used for regulation of foods and drugs, Anal. Chem., 1982, 54, 67A-76A. 